\title{
Oral Phosphorus Supplementation Secondarily Increases Circulating Fibroblast Growth Factor 23 Levels at Least Partially via Stimulation of Parathyroid Hormone Secretion
}

\author{
Satoshi TAKasugi, Miho Akutsu and Masashi Nagata \\ Food Science Research Laboratories, Division of Research and Development, Meiji Co., Ltd. \\ 540 Naruda, Odawara, Kanagawa 250-0862, Japan
}

(Received August 20, 2013)

\begin{abstract}
Summary Oral phosphorus supplementation stimulates fibroblast growth factor 23 (FGF23) secretion; however, the underlying mechanism remains unclear. The aim of this study was to investigate the involvement of parathyroid hormone (PTH) in increased plasma FGF23 levels after oral phosphorus supplementation in rats. Rats received single dose of phosphate with concomitant subcutaneous injection of saline or human PTH (1-34) after treatment with cinacalcet or its vehicle. Cinacalcet is a drug that acts as an allosteric activator of the calcium-sensing receptor and reduces PTH secretion. Plasma phosphorus and PTH levels significantly increased $1 \mathrm{~h}$ after oral phosphorus administration and returned to basal levels within $3 \mathrm{~h}$, while plasma FGF23 levels did not change up to $2 \mathrm{~h}$ post-treatment, but rather significantly increased at $3 \mathrm{~h}$ after administration and maintained higher levels for at least $6 \mathrm{~h}$ compared with the 0 time point. Plasma PTH and FGF23 levels were significantly lower in the cinacalcet-treated rats than in the vehicle-treated rats. Plasma phosphorus levels were significantly higher in the cinacalcet-treated rats than in the vehicle-treated rats at 2, 3, 4, and $6 \mathrm{~h}$ after oral phosphorus administration. Furthermore, rats treated with cinacalcet+human PTH (1-34) showed transiently but significantly higher plasma FGF23 levels at $3 \mathrm{~h}$ after oral phosphorus administration compared with cinacalcet-treated rats. These results suggest that oral phosphorus supplementation secondarily increases circulating FGF23 levels at least partially by stimulation of PTH secretion.
\end{abstract}

Key Words parathyroid hormone, fibroblast growth factor 23, cinacalcet, phosphorus supplementation

Phosphorus is an essential nutrient for many biological functions, including cellular signal transduction, mineral metabolism, and energy exchange. On the other hand, a relatively high serum phosphorus level is reportedly a risk factor for cardiovascular disease $(1,2)$ because inorganic phosphates have been shown to stimulate differentiation of vascular smooth muscle cells into calcifying vascular cells (3). Therefore, serum phosphorus regulation may help to prevent cardiovascular diseases.

Fibroblast growth factor 23 (FGF23) is a bone-derived hormone that plays a regulatory role in phosphorus homeostasis. FGF23 has been shown to reduce serum phosphorus levels by inhibition of proximal tubular phosphorus reabsorption through decreased expression of types $2 \mathrm{a}$ and $2 \mathrm{c}$ sodium-phosphate cotransporters and by decreasing intestinal phosphorus absorption through inhibiting calcitriol synthesis in the kidney (4). Moreover, FGF23-null mice were shown to exhibit severe vascular calcification with concomitant hyperphosphatemia (5). These facts suggest that FGF23 may have a protective effect on vascular calcification by hyperphosphatemia suppression. In contrast, several hemodialysis

E-mail: satoshi.takasugi@meiji.com studies reported that elevated FGF23 levels were associated with aortic calcification (6) and mortality (7, 8 ) independent of serum phosphorus levels. Elevated serum FGF23 levels, even within the normal range, are reportedly associated with increased left ventricular mass index and increased risk for the presence of left ventricular hypertrophy in elderly subjects (9). Furthermore, a recent study suggested that elevated FGF23 levels contributed directly to high rates of left ventricular hypertrophy and mortality in individuals with chronic kidney diseases (10). Thus, FGF23 regulation may also play a clinically significant role in the prevention of cardiovascular diseases.

Oral phosphorus supplementation is known to stimulate FGF23 secretion, although the underlying mechanism has not been clarified. In our preliminary study, we found that plasma FGF23 levels in rats increased after oral phosphorus administration at a physiological dose; however, this increase slowly began around the time that plasma phosphorus levels had decreased (unpublished data). Based on these findings, we reasoned that oral phosphorus supplementation may secondarily increase FGF23 secretion. Parathyroid hormone (PTH) is also secreted in response to phosphorus administration (11) and subsequently increases urinary phosphorus excre- 
tion. López et al. (12) recently reported that plasma FGF23 levels in parathyroidectomized rats were lower than those in sham rats, but dose-dependently recovered with continuous PTH administration, which suggested that PTH stimulated FGF23 secretion. Therefore, we hypothesized that oral phosphorus supplementation secondarily increases FGF23 secretion via stimulation of PTH secretion. The aim of this study was to investigate PTH involvement in the increase in plasma FGF23 levels after oral phosphorus administration in rats. We provide evidence that oral phosphorus supplementation secondarily increases circulating FGF23 levels at least partially by stimulation of PTH secretion.

\section{Materials and Methods}

Animals. Eighteen female Wistar rats were purchased from Japan SLC, Inc. (Shizuoka, Japan) and cared for in accordance with the guidelines of the ethics committee on animal use of Meiji Co., Ltd. The rats were housed in plastic cages in a temperature-, humidity-, and light-controlled room $\left(21 \pm 2{ }^{\circ} \mathrm{C}, 55 \pm 15 \%\right.$ humidity, 12-h light/dark cycle) and given ad libitum access to a standard diet (AIN-93M; 0.5\% calcium and 0.3\% phosphorus) (13).

Study design. After acclimation, the rats (12 wk old) were divided into three body weight-matched groups of six rats each: a control group, a cinacalcet (CIN) group, and a CIN+human PTH (hPTH) (1-34) group. CIN is a drug that acts as an allosteric activator of the calcium-sensing receptor (CaSR) and reduces PTH levels by increasing the sensitivity of the parathyroid gland CaSR to ambient calcium $(14,15)$. PTH (1-34) is a bioactive N-terminal 34-amino acid fragment of hPTH. After overnight fasting, the CIN and CIN + hPTH (1-34) groups were orally administered CIN hydrochloride (Regpara; Kyowa Hakko Kirin, Co., Ltd., Tokyo, Japan) at a dose of $30 \mathrm{mg} / \mathrm{kg}$ and the control group was administered a vehicle only (20\% hydroxypropyl- $\beta$-cyclodextrin). After $30 \mathrm{~min}$, all rats were orally administered $90 \mathrm{mg} / \mathrm{kg}$ of phosphorus as a $40-\mathrm{mg} / \mathrm{mL}$ potassium dihydrogen phosphate solution. Immediately after phosphorus administration, the $\mathrm{CIN}+\mathrm{hPTH}$ group subcutaneously received a synthetic hPTH (1-34) substitute (teriparatide acetate, $80 \mu \mathrm{g} / \mathrm{kg}$; Teribone; Asahi Kasei Pharma Corp., Tokyo, Japan), whereas the control and CIN groups received physiological saline. The dose of phosphorus was equivalent to approximately one-third of the daily phosphorus intake of rats. The dose of CIN was determined based on the previous studies $(16,17)$, which showed that oral CIN administration at $30 \mathrm{mg} / \mathrm{kg}$ substantially decreased serum levels of intact PTH until 12 or $24 \mathrm{~h}$ after administration in normal rats. The dose of hPTH (1-34) was determined based on the study of $\mathrm{Li}$ et al. (18), which reported that normal rats receiving subcutaneous injection of hPTH (1-34) at $80 \mu \mathrm{g} /$ $\mathrm{kg}$ showed approximately $50 \mathrm{pg} / \mathrm{mL}$ of serum hPTH (1-34) levels at $1 \mathrm{~h}$ after injection.

Blood samples were collected from the tail vein using heparinized capillary tubes immediately before and at 1 , $2,3,4$, and $6 \mathrm{~h}$ after oral phosphorus administration.
The samples were centrifuged at 11,000 rpm for $5 \mathrm{~min}$ and the plasma portion was then stored at $-80^{\circ} \mathrm{C}$ until being assayed. These samples were used to determine the levels of phosphorus, calcium, intact PTH, FGF23, and hPTH (1-34). After the experiment, rats were euthanized by exsanguination through the aorta under pentobarbital anesthesia.

Measurements of plasma phosphorus, calcium, intact PTH, FGF23, and hPTH (1-34). Plasma phosphorus and calcium levels were measured using commercial kits (Wako Pure Chemical Industries, Ltd., Osaka, Japan). Plasma FGF23 levels was determined using the FGF23 enzyme-linked immunosorbent assay (ELISA) kit (Kainos Laboratories, Tokyo, Japan). Levels of plasma intact PTH were assayed using the Rat Bioactive Intact PTH ELISA kit (Immunotopics International, San Clemente, CA), which is specific for the full-length intact 1-84 form of the molecule. Due to insufficient volume, plasma samples from each group were pooled and then assayed for plasma hPTH (1-34) levels using the High Sensitivity Human PTH (1-34) ELISA kit (Immunotopics International).

Statistical analysis. Values are presented as the mean Istandard error of the mean (SE). Bartlett's test was performed to determine the homogeneity of variances. If the assumption of homogeneity of variances was not rejected, differences among groups at each time point were analyzed using the analysis of variance (ANOVA) followed by the Tukey-Kramer test and differences between time point 0 and each time point within each group were analyzed by ANOVA followed by Dunnett's test. If the assumption was rejected, differences at each time point were analyzed using the Kruskal-Wallis test followed by the Steel-Dwass multiple comparison test and differences between time point 0 and each time point within each group were analyzed using the Kruskal-Wallis test followed by Steel's test. All statistical analyses were performed using Ekuseru-Toukei software (Social Survey Research Information Co., Ltd., Tokyo, Japan, 2012). A probability ( $p$ ) value $<0.05$ was considered statistically significant.

\section{Results and Discussion}

To investigate PTH involvement in the increase in plasma FGF23 levels after oral phosphorus administration, we orally administered phosphorus with concomitant subcutaneous injection of saline or hPTH (1-34) following treatment with CIN or its vehicle in rats. Plasma phosphorus levels significantly increased $1 \mathrm{~h}$ after oral phosphorus administration, but returned to basal levels within $3 \mathrm{~h}$ (Fig. 1). Plasma PTH levels significantly increased $1 \mathrm{~h}$ after oral phosphorus administration and returned to basal levels within $3 \mathrm{~h}$ as did plasma phosphorus levels (Fig. 2). However, plasma FGF23 levels did not change up to $2 \mathrm{~h}$ post-treatment, but rather increased at $3 \mathrm{~h}$ after oral phosphorus administration and maintained higher levels for at least $6 \mathrm{~h}$ compared with the 0 time point (Fig. 3). Nishida et al. (11) reported that serum FGF23 levels did not change over a short period (up to $6 \mathrm{~h}$ ), but slowly increased at 


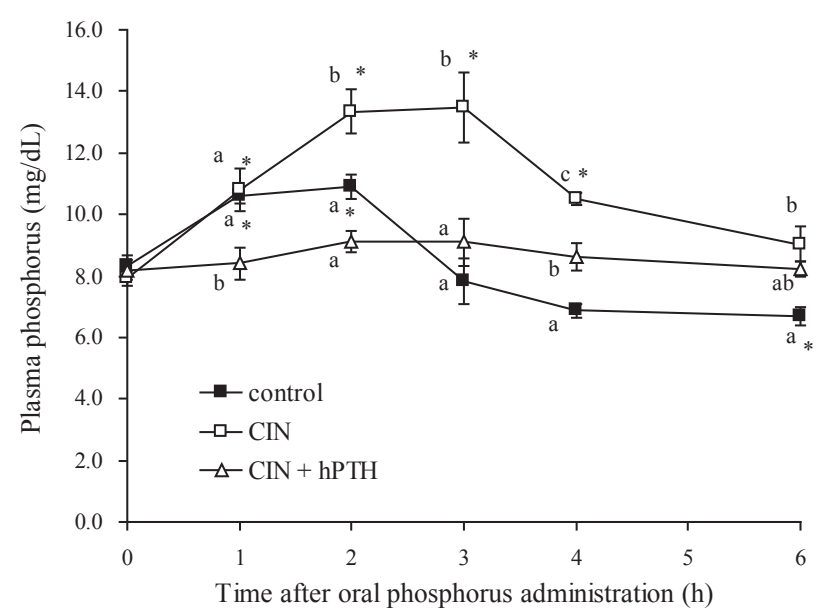

Fig. 1. Effects of cinacalcet (CIN) administration with or without human parathyroid hormone (hPTH) injection on plasma phosphorus levels after oral phosphorus administration. Values are presented as the mean \pm SE. Values with different letters at each time point are significantly different $(p<0.05)$. Values with asterisks indicate significant differences $(p<0.05)$ compared to before oral phosphorus administration (time point 0 ) in each group.

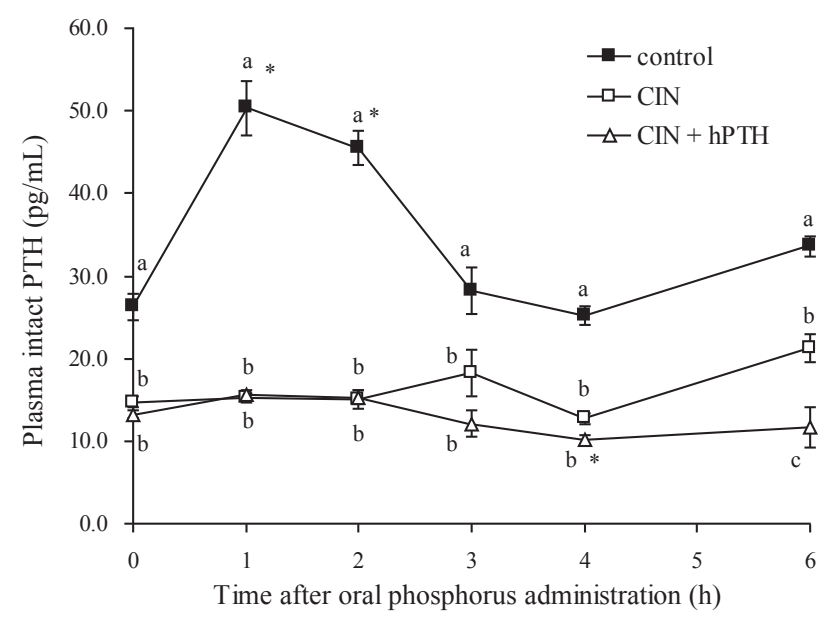

Fig. 2. Effects of cinacalcet (CIN) administration with or without human parathyroid hormone (hPTH) injection on plasma intact PTH levels after oral phosphorus administration. Values are presented as the mean \pm SE. Values with different letters at each time point are significantly different $(p<0.05)$. Values with asterisks indicate significant differences $(p<0.05)$ compared to before oral phosphorus administration (time point 0 ) in each group.

$8 \mathrm{~h}$ after oral phosphorus administration at a dose of $1,200 \mathrm{mg}$ in healthy men, while serum phosphorus and PTH levels increased over a short time, which is consistent with our findings. Oral phosphorus administration did not affect plasma calcium levels (Fig. 4). PTH secretion is primarily controlled by CaSR in response to extracellular calcium (19). In contrast, our results indicated that oral phosphorus administration stimulated PTH secretion independently of plasma calcium levels.

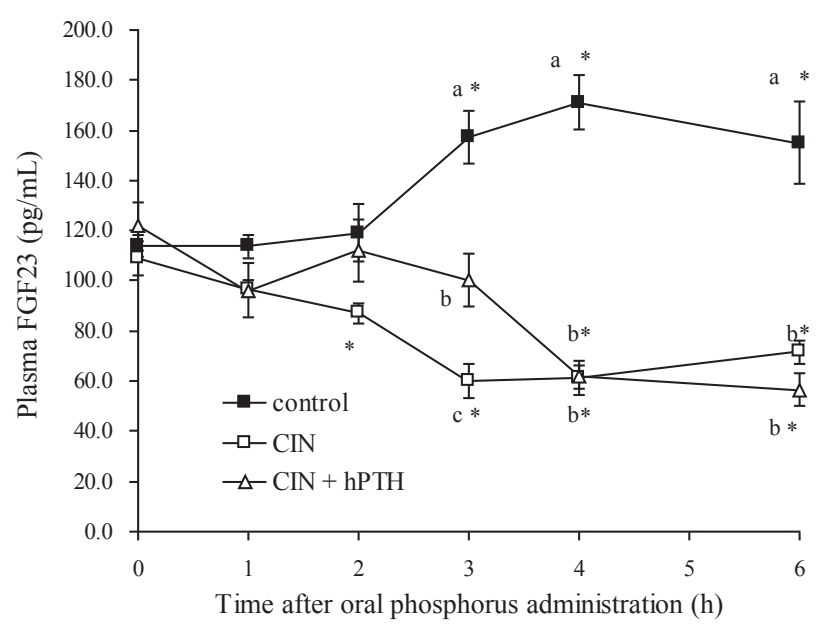

Fig. 3. Effects of cinacalcet (CIN) administration with or without human parathyroid hormone (hPTH) injection on plasma fibroblast growth factor 23 (FGF23) levels after oral phosphorus administration. Values are presented as the mean \pm SE. Values with different letters at each time point are significantly different $(p<0.05)$. Values with asterisks indicate significant differences $(p<0.05)$ compared to before oral phosphorus administration (time point 0 ) in each group.

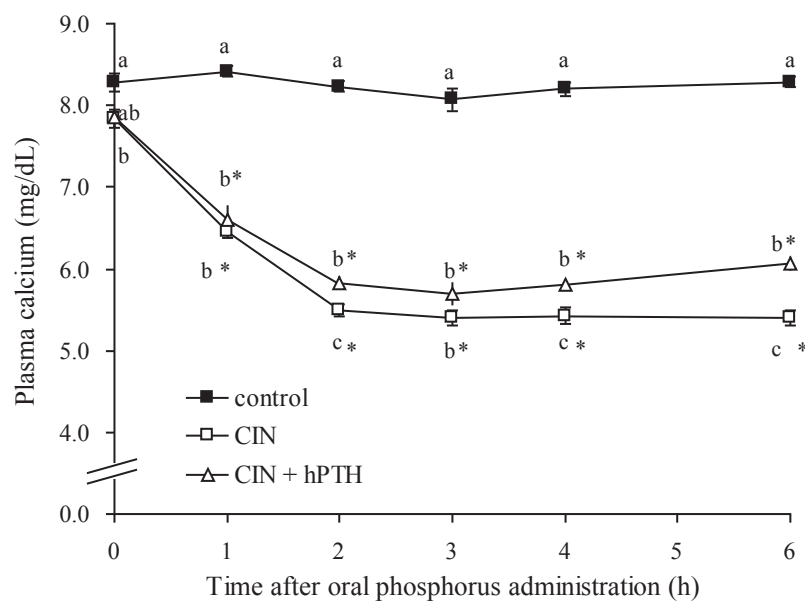

Fig. 4. Effects of cinacalcet (CIN) administration with or without human parathyroid hormone (hPTH) injection on plasma calcium levels after oral phosphorus administration. Values are presented as the mean \pm SE. Values with different letters at each time point are significantly different $(p<0.05)$. Values with asterisks indicate significant differences $(p<0.05)$ compared to before oral phosphorus administration (time point 0 ) in each group.

Several in vitro (20-22) and in vivo (23-25) studies have demonstrated that phosphorus administration can stimulate PTH secretion independently of calcium levels, which was in accordance with our results.

Treatment with CIN significantly inhibited the increase in plasma PTH levels (Fig. 2). Plasma hPTH (1-34) levels in rats treated with $\mathrm{CIN}+\mathrm{hPTH}(1-34)$ were $102.6 \mathrm{pg} / \mathrm{mL}(1 \mathrm{~h}), 28.4 \mathrm{pg} / \mathrm{mL}(2 \mathrm{~h})$ and $8.9 \mathrm{pg} /$ $\mathrm{mL}(3 \mathrm{~h})$ and decreased to non-detectable levels at $4 \mathrm{~h}$. 
In CIN-treated rats, plasma phosphorus levels were significantly higher than those in rats without CIN treatment at 2, 3, 4, and $6 \mathrm{~h}$ after oral phosphorus administration (Fig. 1). In addition, rats treated with $\mathrm{CIN}+\mathrm{hPTH}$ (1-34) showed significantly lower plasma phosphorus levels at 1, 2, 3, and $4 \mathrm{~h}$ after oral phosphorus administration compared to CIN-treated rats. These results indicated that PTH suppressed the increase in plasma phosphorus levels after oral phosphorus administration. Plasma FGF23 levels in CIN-treated rats decreased until $3 \mathrm{~h}$ after oral phosphorus administration and remained low for at least $6 \mathrm{~h}$ post-treatment compared with the 0 time point. Furthermore, rats treated with CIN + hPTH (1-34) showed transiently but significantly higher plasma FGF23 levels at $3 \mathrm{~h}$ after oral phosphorus administration compared to CIN-treated rats. These findings suggested that oral phosphorus administration secondarily increased plasma FGF23 levels at least partially via stimulation of PTH secretion. Some researchers reported that PTH increased FGF23 mRNA levels in vitro (26) and circulating FGF23 levels in vivo (12), which supports our results. Our findings also suggested that elevated plasma phosphorus levels per se did not stimulate FGF23 secretion as suggested previously (27).

In the present study, hPTH (1-34) administration in CIN-treated rats partially recovered the decrease in plasma FGF23 levels, which could be explained by the following two possibilities: first, plasma hPTH (1-34) levels increased in a transient manner, which may not have been sufficient to continuously recover the decrease in plasma FGF23 levels; second, CIN treatment inhibited the increase in plasma FGF23 levels by another mechanism in addition to inhibition of PTH secretion. Rodriguez-Ortiz et al. (28) reported that hypocalcemia reduced circulating FGF23 levels. In the present study, plasma calcium levels in CIN-treated rats continued to decrease until $2 \mathrm{~h}$ after oral phosphorus administration and afterward, they remained lower for at least $6 \mathrm{~h} \mathrm{com-}$ pared with the 0 time point (Fig. 4). In addition, hPTH (1-34) treatment only partially recovered the decrease in plasma calcium levels induced by CIN treatment, although hPTH (1-34) administration at the same dose used in the present study has been reported to effectively increase plasma calcium levels in rats (18). These findings suggested that CIN treatment may have decreased plasma FGF23 levels by decreasing plasma calcium levels and by decreasing PTH secretion; thus the inhibitory effects of hypocalcemia on FGF23 secretion may have overwhelmed the stimulatory effect of hPTH (1-34), which supports the proposition by Rodriguez-Ortiz et al. (28) that hypocalcemia limits phosphorus-induced FGF23 production. Nemeth et al. (16) reported that CIN administration at the same dose used in the present study not only decreased serum PTH levels but also increased serum calcitonin levels in rats. Calcitonin is a hormone that lowers serum calcium levels. In the present study, CIN treatment seemed to decrease plasma calcium levels by inducing calcitonin and decreasing PTH secretion.

PTH is known to induce calcitriol synthesis. Some researchers reported that calcitriol stimulates FGF23 synthesis in the calvarium of rats (29) and increases circulating FGF23 levels in thyroparathyroidectomized rats (30). These reports collectively indicate that PTH may indirectly stimulate FGF23 secretion by stimulation of calcitriol synthesis. On the other hand, López et al. (12) reported that PTH can also modify FGF23 under conditions in which calcitriol is controlled. Furthermore, PTH was reported to increase FGF23 mRNA levels in vitro (26). Further studies are required to clarify whether the stimulatory effects of PTH on FGF23 secretion depend on calcitriol synthesis.

Elevated FGF23 levels were reportedly associated with aortic calcification (6) and mortality $(7,8)$ independent of serum phosphorus levels in patients with hemodialysis, and with increased left ventricular mass index and increased risk for the presence of left ventricular hypertrophy in elderly subjects (9). In addition, a recent study suggested that elevated FGF23 levels contributed directly to high rates of left ventricular hypertrophy and mortality in individuals with chronic kidney diseases (10). These reports suggest that FGF23 regulation may play a clinically significant role in the prevention of cardiovascular diseases. In the present study, we demonstrated that phosphorus-induced elevation of FGF23 at least partially resulted from PTH secretion. Thus, regulation of PTH might help to maintain normal levels of FGF23, leading to the prevention of cardiovascular diseases. In conclusion, our findings suggest that oral phosphorus supplementation secondarily increases circulating FGF23 levels at least partially by stimulation of PTH secretion.

\section{Acknowledgments}

The authors sincerely thank Yasuko Suetake (World Intec) for expert technical assistance.

\section{REFERENCES}

1) Dhingra R, Sullivan LM, Fox CS, Wang TJ, D'Agostino RB Sr, Gaziano JM, Vasan RS. 2007. Relations of serum phosphorus and calcium levels to the incidence of cardiovascular disease in the community. Arch Intern Med 167: 879-885.

2) Tonelli M, Sacks F, Pfeffer M, Gao Z, Curhan G. 2005. Relation between serum phosphate level and cardiovascular event rate in people with coronary disease. Circulation 112: 2627-2633.

3) Jono S, McKee MD, Murry CE, Shioi A, Nishizawa Y, Mori K, Morii H, Giachelli CM. 2000. Phosphate regulation of vascular smooth muscle cell calcification. Circ Res 87: E10-17.

4) Shimada T, Hasegawa H, Yamazaki Y, Muto T, Hino R, Takeuchi Y, Fujita T, Nakahara K, Fukumoto S, Yamashita T. 2004. FGF-23 is a potent regulator of vitamin D metabolism and phosphate homeostasis. J Bone Miner Res 19: 429-435.

5) Memon F, El-Abbadi M, Nakatani T, Taguchi T, Lanske B, Razzaque MS. 2008. Does Fgf23-klotho activity influence vascular and soft tissue calcification through regulating mineral ion metabolism? Kidney Int 74: 566-570.

6) Nasrallah MM, El-Shehaby AR, Salem MM, Osman NA, El Sheikh E, Sharaf El Din UA. 2010. Fibroblast growth 
factor-23 (FGF-23) is independently correlated to aortic calcification in haemodialysis patients. Nephrol Dial Transplant 25: 2679-2685.

7) Gutiérrez OM, Mannstadt M, Isakova T, Rauh-Hain JA, Tamez H, Shah A, Smith K, Lee H, Thadhani R, Jüppner H, Wolf M. 2008. Fibroblast growth factor 23 and mortality among patients undergoing hemodialysis. N Engl J Med 359: 584-592.

8) Jean G, Terrat JC, Vanel T, Hurot JM, Lorriaux C, Mayor B, Chazot C. 2009. High levels of serum fibroblast growth factor (FGF)-23 are associated with increased mortality in long haemodialysis patients. Nephrol Dial Transplant 24: 2792-2796.

9) Mirza MA, Larsson A, Melhus H, Lind L, Larsson TE. 2009. Serum intact FGF23 associate with left ventricular mass, hypertrophy and geometry in an elderly population. Atherosclerosis 207: 546-551.

10) Faul C, Amaral AP, Oskouei B, Hu MC, Sloan A, Isakova T, Gutiérrez OM, Aguillon-Prada R, Lincoln J, Hare JM, Mundel P, Morales A, Scialla J, Fischer M, Soliman EZ, Chen J, Go AS, Rosas SE, Nessel L, Townsend RR, Feldman HI, St John Sutton M, Ojo A, Gadegbeku C, Di Marco GS, Reuter S, Kentrup D, Tiemann K, Brand M, Hill JA, Moe OW, Kuro-O M, Kusek JW, Keane MG, Wolf M. 2011. FGF23 induces left ventricular hypertrophy. J Clin Invest 121: 4393-4408.

11) Nishida $Y$, Taketani $Y$, Yamanaka-Okumura H, Imamura F, Taniguchi A, Sato T, Shuto E, Nashiki K, Arai H, Yamamoto H, Takeda E. 2006. Acute effect of oral phosphate loading on serum fibroblast growth factor 23 levels in healthy men. Kidney Int 70: 2141-2147.

12) López I, Rodríguez-Ortiz ME, Almadén Y, Guerrero F, de Oca AM, Pineda C, Shalhoub V, Rodríguez M, AguileraTejero E. 2011. Direct and indirect effects of parathyroid hormone on circulating levels of fibroblast growth factor 23 in vivo. Kidney Int 80: 475-482.

13) Reeves PG, Nielsen FH, Fahey GC Jr. 1993. AIN-93 purified diets for laboratory rodents: final report of the American Institute of Nutrition ad hoc writing committee on the reformulation of the AIN-76A rodent diet. J Nutr 123: 1939-1951.

14) Fox J, Lowe SH, Conklin RL, Nemeth EF. 1999. The calcimimetic NPS R-568 decreases plasma PTH in rats with mild and severe renal or dietary secondary hyperparathyroidism. Endocrine 10: 97-103.

15) Nemeth EF, Steffey ME, Hammerland LG, Hung BC, Van Wagenen BC, DelMar EG, Balandrin MF. 1998. Calcimimetics with potent and selective activity on the parathyroid calcium receptor. Proc Natl Acad Sci USA 95: 4040-4045.

16) Nemeth EF, Heaton WH, Miller M, Fox J, Balandrin MF, Van Wagenen BC, Colloton M, Karbon W, Scherrer J, Shatzen E, Rishton G, Scully S, Qi M, Harris R, Lacey D, Martin D. 2004. Pharmacodynamics of the type II calcimimetic compound cinacalcet HCl. J Pharmacol Exp Ther 308: $627-635$.

17) Rodriguez M, Nemeth E, Martin D. 2005. The calciumsensing receptor: a key factor in the pathogenesis of secondary hyperparathyroidism. Am J Physiol Renal Physiol 288: F253-F264.
18) Li X, Liu H, Qin L, Tamasi J, Bergenstock M, Shapses S, Feyen JH, Notterman DA, Partridge NC. 2007. Determination of dual effects of parathyroid hormone on skeletal gene expression in vivo by microarray and network analysis. J Biol Chem 282: 33086-33097.

19) Thomsen AR, Smajilovic S, Bräuner-Osborne H. 2012. Novel strategies in drug discovery of the calcium-sensing receptor based on biased signaling. Curr Drug Targets 13: 1324-1335.

20) Almaden Y, Canalejo A, Hernandez A, Ballesteros E, Garcia-Navarro S, Torres A, Rodriguez M. 1996. Direct effect of phosphorus on PTH secretion from whole rat parathyroid glands in vitro. J Bone Miner Res 11: 970-976.

21) Nielsen PK, Feldt-Rasmussen U, Olgaard K. 1996. A direct effect in vitro of phosphate on PTH release from bovine parathyroid tissue slices but not from dispersed parathyroid cells. Nephrol Dial Transplant 11: 1762-1768.

22) Slatopolsky E, Finch J, Denda M, Ritter C, Zhong M, Dusso A, MacDonald PN, Brown AJ. 1996. Phosphorus restriction prevents parathyroid gland growth. High phosphorus directly stimulates PTH secretion in vitro. $J$ Clin Invest 97: 2534-2540.

23) Hernández A, Concepción MT, Rodríguez M, Salido E, Torres A. 1996. High phosphorus diet increases preproPTH mRNA independent of calcium and calcitriol in normal rats. Kidney Int 50: 1872-1878.

24) Kilav R, Silver J, Naveh-Many T. 1995. Parathyroid hormone gene expression in hypophosphatemic rats. J Clin Invest 96: 327-333.

25) Estepa JC, Aguilera-Tejero E, López I, Almaden Y, Rodriguez M, Felsenfeld AJ. 1999. Effect of phosphate on parathyroid hormone secretion in vivo. J Bone Miner Res 14: 1848-1854.

26) Lavi-Moshayoff V, Wasserman G, Meir T, Silver J, NavehMany T. 2010. PTH increases FGF23 gene expression and mediates the high-FGF23 levels of experimental kidney failure: a bone parathyroid feedback loop. Am J Physiol Renal Physiol 299: F882-F889.

27) Liu S, Tang W, Zhou J, Stubbs JR, Luo Q, Pi M, Quarles LD. 2006. Fibroblast growth factor 23 is a counter-regulatory phosphaturic hormone for vitamin D. J Am Soc Nephrol 17: 1305-1315.

28) Rodriguez-Ortiz ME, López I, Muñoz-Castañeda JR, Martinez-Moreno JM, Ramírez AP, Pineda C, Canalejo A, Jaeger P, Aguilera-Tejero E, Rodriguez M, Felsenfeld A, Almaden Y. 2012. Calcium deficiency reduces circulating levels of FGF23. J Am Soc Nephrol 23: 1190-1197.

29) Saji F, Shigematsu T, Sakaguchi T, Ohya M, Orita H, Maeda Y, Ooura M, Mima T, Negi S. 2010. Fibroblast growth factor 23 production in bone is directly regulated by 1 \{alpha\},25-dihydroxyvitamin D, but not PTH. Am J Physiol Renal Physiol 299: F1212-F1217.

30) Saito H, Maeda A, Ohtomo S, Hirata M, Kusano K, Kato S, Ogata E, Segawa H, Miyamoto K, Fukushima N. 2005. Circulating FGF-23 is regulated by 1alpha,25-dihydroxyvitamin D3 and phosphorus in vivo. J Biol Chem 280: 2543-2549. 